\title{
Prevalencia de presentación de algunos agentes zoonóticos transmitidos por caninos y felinos en Medellín, Colombia
}

\author{
Laura Castrillón-Salazar ${ }^{10}$ M.Sc, Laura López-Diez ${ }^{10}$ MV, Raul Sanchez-Nodarse ${ }^{2}$ MV

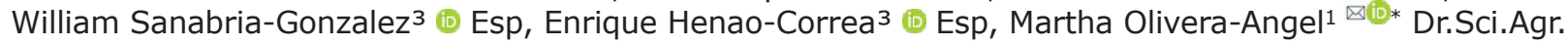 \\ 1 Universidad de Antioquia, Facultad de Ciencias Agrarias, Grupo de Investigación Biogénesis. Medellín, Colombia. \\ 2 Universidad de Antioquia, Facultad de Ciencias Agrarias. Medellín, Colombia. \\ ${ }^{3}$ Alcaldía de Medellín, Área Técnica de Prevención y Control de Zoonosis. Medellín, Colombia. \\ *Correspondencia: martha.olivera@udea.edu.co
}

Recibido: Febrero 2018; Aceptado: Diciembre 2018; Publicado: Febrero 2019.

\section{RESUMEN}

Objetivo. Determinar la prevalencia de agentes zoonóticos en caninos y felinos en Medellín, Colombia. Materiales y métodos. Se muestrearon 1501 individuos para el análisis de parásitos gastrointestinales zoonóticos por medio de coprología directa y flotación. Se examinaron 500 sueros caninos por medio de PARP-2ME y MAT para el diagnóstico de Brucella canis y Leptospira sp, respectivamente. Se procesaron 500 sueros felinos por medio de IFI para el diagnóstico de Toxoplasma gondii. Se estableció la frecuencia para cada zoonosis y la significancia estadística para las diferentes variables ( $\mathrm{p} \leq 0.05$; OR $\geq 1$; IC 95\%). Resultados. El $23.6 \%$ de los caninos y $16.3 \%$ de los felinos fueron positivos a parásitos gastrointestinales, siendo los Ancylostomideos y $D$. caninum los más prevalente, respectivamente; la especie, edad, sexo, sector, estrato socioeconómico y el mes de muestreo presentaron asociaciones con el parasitismo gastrointestinal en mascotas. En caninos se evidenció una seroprevalencia del $6.6 \%$ para B. canis y $8.4 \%$ para Leptospira sp; en felinos del $56.2 \%$ para $T$. gondii. Todas las anteriores asociadas con la zona de muestreo, mes, edad y estrato. Conclusiones. Las mascotas ubicadas en diferentes comunas y estratos socioeconómicos con condiciones de calidad de vida menores representan un riesgo de transmisión zoonótica.

Palabras clave: Brucelosis canina, leptospirosis, mascotas, parasitismo, toxoplasmosis (Fuente: DeCS).

\begin{abstract}
Objective. To determine in Medellin, Colombia, the prevalence of zoonotic agents in canines and felines. Materials and methods. 1501 individuals were sampled for the analysis of zoonotic gastrointestinal parasites by direct coprology and flotation. 500 canine sera were examined by PARP-2ME and MAT for the diagnosis of Brucella canis and Leptospira sp, respectively. 500 feline sera were processed by IFI for the diagnosis of Toxoplasma gondii. The frequency for each zoonosis and the statistical significance for the different variables were established ( $p \leq 0.05 ; O R \geq 1 ; 95 \% \mathrm{CI}$ ). Results. $23.6 \%$ of canines and $16.3 \%$ of felines were positive for gastrointestinal parasites; respectively Ancylostomids and $D$. caninum were the most prevalent; species, age, sex, sector, socioeconomic status and the month of sampling showed associations with gastrointestinal parasitism in pets. Canines showed a seroprevalence of $6.6 \%$ in B. canis and $8.4 \%$, Leptospira sp; in felines $56.2 \%$ for T. gondii. All of the above associated with the commune, month of sampling, age and stratum. Conclusions. Pets located in different communes and socioeconomic strata with lower quality of life conditions represent a risk of zoonotic transmission.
\end{abstract}

Keywords: Canine brucellosis, leptospirosis, pets, parasitism, toxoplasmosis (Source: DeCS).

Como citar (Vancouver)

Castrillón-Salazar L, López-Diez L, Sanchez-Nodarse R, Sanabria-Gonzalez W, Henao-Correa E, Olivera-Angel M. Prevalencia de presentación de algunos agentes zoonóticos transmitidos por caninos y felinos en Medellín, Colombia. Rev MVZ Cordoba. 2019; 24(1):7119-7126. DOI: https://doi.org/10.21897/rmvz.1524 cc) (i) (2) Attribution 4.0 (https://creativecommons.org/licenses/by-sa/4.0/), que permite el uso sin restricciones, la distribución y la reproducción en cualquier medio, siempre que se otorgue el crédito apropiado al autor o autores originales y la fuente. 


\section{INTRODUCCIÓN}

La OMS establece que el $60-65 \%$ de las enfermedades que afectan al humano son de carácter zoonótico. La población infantil es la más expuesta a infectarse por el contacto con los caninos y felinos debido a la exposición mediante el juego y la convivencia con éstos; también por los animales que deambulan libremente o porque los propietarios desconocen los planes de prevención $(\underline{1}, \underline{2}, \underline{3})$.

Medellín, una de las ciudades con mayor crecimiento $(0.9 \%)$, para el año 2016 contaba con una población de 2.486.723 personas; se estima que la población de mascotas aumentará proporcionalmente a la población humana, factor determinante en los proyectos de salud pública para el manejo, prevención y control (4). Para el 2015 el $25.5 \%$ de la población tenía perros y el $10.7 \%$ gatos; adicionalmente, el $50 \%$ de los caninos se encuentran en el Área Metropolitana, con presencia de perros doméstico-callejeros que circulan libremente $(\underline{1}, \underline{4})$.

Los parásitos gastrointestinales de caninos y felinos pueden generar enfermedades en los humanos como Larva Migrans Cutánea u Ocular (LMC, LMO), o alteraciones gastrointestinales en niños y adultos $(\underline{2}, \underline{5}, \underline{6})$. La brucelosis canina, leptospirosis y toxoplasmosis cursan de forma subclínica, inespecífica o agudas tanto en animales como en el hombre, llegando a generar en éste último cuadros clínicos severos $(\underline{7}, \underline{8}, \underline{9}, \underline{10})$. Las mascotas son los principales mediadores de contaminación en zonas de esparcimiento familiar por medio de las excretas, por contacto con secreciones animales o por contaminación de alimentos $(\underline{6}, \underline{10})$.

El objetivo del estudio fue establecer la prevalencia de estos agentes en los animales de compañía en Medellín, con el fin de aportar a la sanidad de las mascotas y disminuir el riesgo de zoonosis.

\section{MATERIALES Y MÉTODOS}

Población y área de estudio. La ciudad de Medellín se ubica a 1479 msnm, cuenta con una extensión de 380.64 $\mathrm{km}^{2}$, una temperatura promedio de $22-24^{\circ} \mathrm{C}$ y humedad relativa de $63-73 \%$; esto con base en información del Instituto de Hidrología, Metrología y Estudios Ambientales (IDEAM) y la Alcaldía de Medellín. Es un estudio de tipo transversal, en el cual se muestrearon caninos y felinos aparentemente sanos, asistentes a las jornadas de esterilización en el año 2016. Se muestrearon 1073 caninos y 428 felinos para parásitos gastrointestinales en las 16 comunas y 5 corregimientos que conforman la ciudad; determinándose por medio de la Encuesta de Calidad de Vida (4) $)$. El número de individuos muestreados para las pruebas de $B$. canis, Leptospira sp. y Toxoplasma gondii fue seleccionado a conveniencia; recolectándose 500 sueros caninos y 500 felinos, distribuidos en 5 comunas periféricas $[1,2,3,8,13]$. Se consignaron datos sobre especies, sexo, edad, comuna, estrato y mes de muestreo. De forma previa, los propietarios firman el consentimiento informado para el uso de las muestras biológicas obtenidas.

Toma de muestras. La materia fecal se recolectó por medio de tacto rectal con previa lubricación con carboximetilcelulosa, se almacenó en frasco tapa rosca, se conservó en formol al $10 \%$ y se refrigeró a $4^{\circ} \mathrm{C}$ hasta su lectura. La muestra de sangre fue colectada en tubo MiniCollet ${ }^{\circledR}$ tapa roja por medio de la canalización de la vena cefálica, fue centrifugada a $1361 \mathrm{~g}$ x 5 min para la separación del suero, el cual fue almacenado en tubo eppendorf de $1.5 \mathrm{ml} \mathrm{a}-20^{\circ} \mathrm{C}$ hasta su proceso.

Diagnóstico parasitológico. El análisis microscópico de materia fecal incluyó los principales agentes causales de zoonosis ( $\underline{5})$. Éste se realizó por medio de dos técnicas, un examen directo con solución salina al $0.85 \%$ y lugol y la técnica de flotación con solución salina saturada descrita por Koffoyd y Barber (11), con el fin de detectar la presencia de huevos o protozoos como Ancylostomideos, Toxocara spp., D. caninum, Giardia spp. y Trichuris spp.

DiagnósticodeBrucella canis. Seusóla Técnica deAglutinación Rápida en Placa con 2 $\beta$-Mercaptoetanol (PARP-2ME) (10). De los 500 sueros se eliminaron tres por hemólisis en la comuna 3.

Diagnóstico Leptospira sp. Se realizó por medio de la Técnica de Microaglutinación (MAT), incluyendo los serovares Canicola, Icterohaemorrhagiae, Grippotyphosa, Pomona, Ballum, Autumnalis, Bratislava y Tarassovi. La técnica implementada y el punto de corte para determinar la seropositividad (títulos de anticuerpos $\geq 1: 100$ ) se establecieron bajo las directrices de la Organización Mundial de Sanidad Animal (OIE) señaladas en el Manual Terrestre de Leptospirosis: capítulo 2.1 .9 y por el Instituto Nacional de Salud de Colombia (INS).

Diagnóstico de Toxoplasma gondii. Se empleó la Técnica de Inmunofluorescencia Indirecta (IFI o IFAT) usando el kit "Toxoplasma gondii IFA Feline IgG Antibody Kit" (Fuller Laboratories. California. USA), siguiendo las indicaciones de la casa comercial.

Análisis estadístico. Para el análisis de los datos fueron empleados los programas estadísticos Excel 2016 ${ }^{\circledR}$, Epidat $3.1^{\circledR}$, SPSS Ver $22^{\circledR}$ con el fin de establecer la asociación y los factores de riesgo para la especie, edad, sexo, comuna, estrato, mes de muestreo y sector, sobre la presencia o ausencia de infección o títulos de las enfermedades estudiadas. Previa agrupación de variables (Tabla 1), se consideró el valor de $p \leq 0.05, O R \geq 10 \leq 1$, IC de $95 \%$ como factor de riesgo o de protección.

Tabla 1. Variables Independientes.

\begin{tabular}{ccc} 
& $1-6$ meses \\
& $7-12$ meses \\
& $13-24$ meses \\
& $25-48$ meses \\
& $\geq 49$ meses \\
\hline Sector & Urbano & Comuna $1-16$ \\
& Rural & Corregimiento $50-90$ \\
\hline \multirow{2}{*}{ Mes de muestreo* } & Periodo Lluvias & Mar - May y Sep - Nov \\
& Periodo Seco & Dic - Feb y Jun - Ago \\
\hline \multirow{2}{*}{ Estrato } & Estrato bajo & 1 y 2 \\
socioeconómico** & Estrato medio & 3 y 4 \\
& Estrato Alto & 5 \\
\hline
\end{tabular}

* Se agrupan con base al IDEAM.

**Se clasifica con base a las características de calidad de vida (4). 
Aspectos éticos. Las muestras de sangre y materia fecal tomadas en las jornadas de esterilización de la Alcaldía de Medellín fueron obtenidas por médicos veterinarios capacitados, con consentimiento informado del propietario y bajo las directrices de la Ley 84 de 1989. El proyecto cuenta con el respectivo aval por parte del Comité de Ética para la Experimentación con Animales (CEEA) de la Universidad de Antioquia mediante el acta No. 113 del 12 de octubre 2017 y la autorización de la Secretaria de Medio Ambiente de la Alcaldía de Medellín.

\section{RESULTADOS}

Parásitos gastrointestinales. En la tabla 2 se encuentran los hallazgos sobre la frecuencia de parásitos gastrointestinales en caninos y felinos, así como la frecuencia para cada agente individual.
Al realizar el análisis comparativo entre caninos y felinos para determinar quiénes representan mayor susceptibilidad ante las mismas infecciones, se encontró que ser felino es un factor de protección ante la positividad a Ancylostomideos $(p=0 ; O R=0.2)$; pero a su vez representan un factor de riesgo para la positividad a Toxocara spp. $(p=0 ; O R=4.4)$ y $D$. caninum $(p=0$; $O R=4.9)$. Los caninos y felinos entre $1-6$ meses $(n=1501)$ fueron encontrados como menos propensos a la afección por Ancylostomideos $(p=0.001 ; O R=0.5)$. El periodo seco se comportó como un factor de riesgo para la afección por $D$. caninum para ambas especies $(n=1501)(p=0.01$; $\mathrm{OR}=10.7$ ).

En la tabla 3 se encuentran los resultados del análisis multivariado, donde se muestran los factores de riesgo o protección para cada especie animal asociados a la positividad del agente parasitario.

Tabla 2. Frecuencias de parásitos gastrointestinales en caninos y felinos por comuna/corregimiento

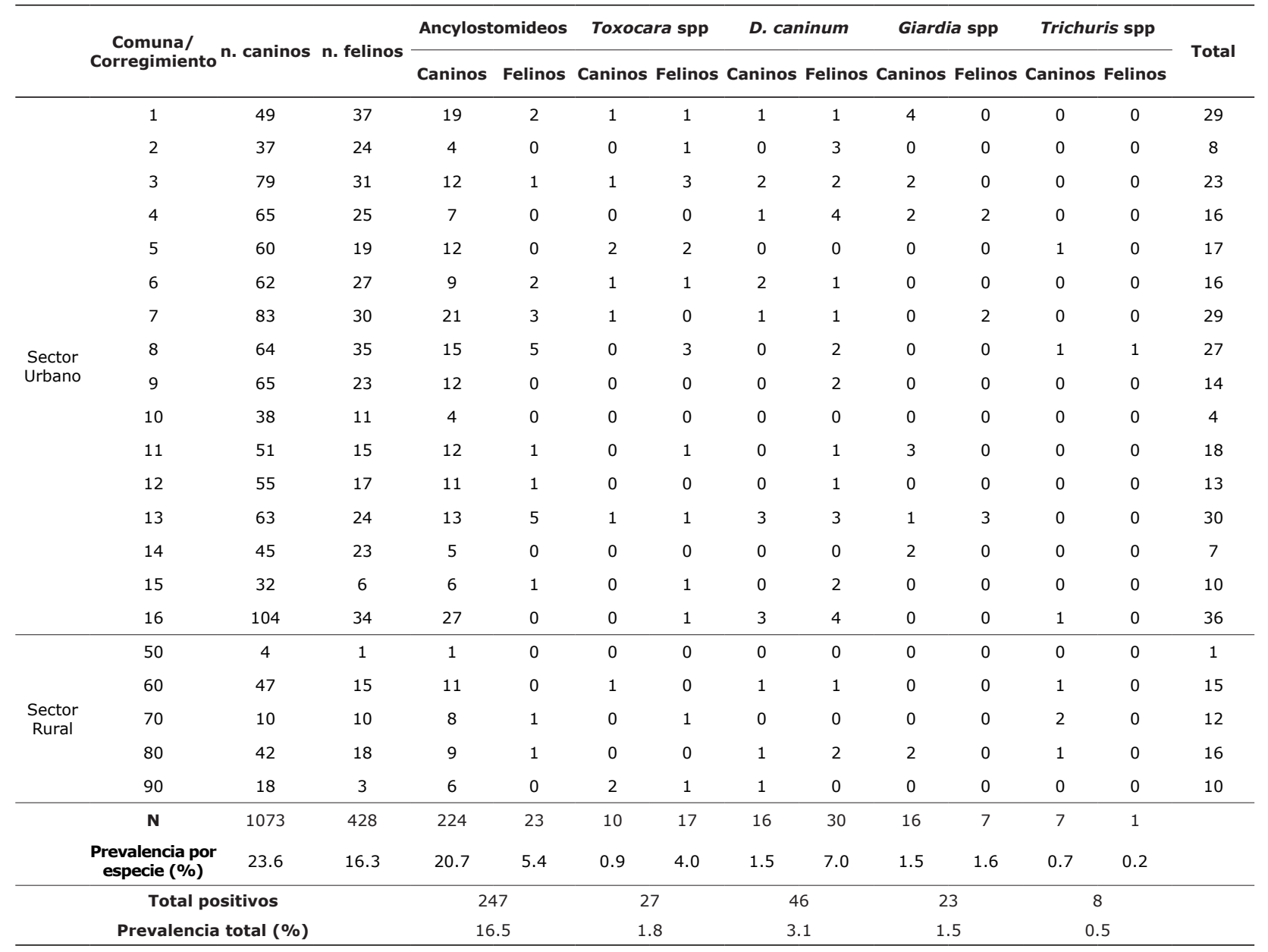

Brucella canis, Leptospira sp. y Toxoplasma gondii. En la tabla 4 se observan los resultados de seropositividad en caninos y felinos para B. canis, Leptospira sp. y $T$. gondii. Para este último se muestran los individuos expuestos ( $\geq 1: 20$ y $\leq 1: 160)$ y los portadores latentes (títulos $\geq 1: 320)$; correspondiendo los últimos a animales aparentemente sanos que albergan el agente, pueden diseminarlo y además son susceptibles de reactivar la infección $(\underline{12}, \underline{13})$. En la tabla 5 se presenta la seroprevalencia para los diferentes serovares de Leptospira sp. En la tabla 6 se encuentran los resultados del análisis multivariado con los factores de riesgo o protección asociados a la seropositividad ante $B$. canis, Leptospira sp. y T. gondii. 
Tabla 3. Análisis bivariado de los factores asociados a la positividad a parásitos gastrointestinales en caninos y felinos. Evidenciando factor de riesgo o factor protector.

\begin{tabular}{|c|c|c|c|c|c|c|c|c|c|c|c|c|c|}
\hline \multicolumn{7}{|c|}{ Asociaciones Parasitismo en Caninos } & \multicolumn{7}{|c|}{ Asociaciones Parasitismo en Felinos } \\
\hline $\begin{array}{c}\text { Variable } \\
\text { Dependiente }\end{array}$ & $\begin{array}{c}\text { Variable } \\
\text { Independiente }\end{array}$ & $\mathbf{x}^{2}$ & $\boldsymbol{p}$ & OR & IC inf & IC sup & $\begin{array}{c}\text { Variable } \\
\text { Dependiente }\end{array}$ & $\begin{array}{c}\text { Variable } \\
\text { Independiente }\end{array}$ & $\mathbf{X}^{2}$ & $\boldsymbol{p}$ & OR & IC inf & IC sup \\
\hline \multirow{10}{*}{$\begin{array}{l}\text { Parasitismo (Todos } \\
\text { los parásitos) }\end{array}$} & Sexo hembras & 15.21 & 0 & 0.6 & 0.42 & 0.75 & \multirow{10}{*}{$\begin{array}{l}\text { Parasitismo } \\
\text { (Todos los } \\
\text { parásitos) }\end{array}$} & \multirow{6}{*}{ Estratos 2} & \multirow{6}{*}{6.88} & \multirow{6}{*}{0} & \multirow{6}{*}{2.2} & \multirow{6}{*}{1.2} & \multirow{6}{*}{3.98} \\
\hline & Estratos 2 & 4.27 & 0.03 & 1.3 & 1.01 & 1.81 & & & & & & & \\
\hline & Estratos 3 & 6.59 & 0.01 & 0.6 & 0.44 & 0.89 & & & & & & & \\
\hline & Comuna 1 & 12.95 & 0 & 2.8 & 1.56 & 5 & & & & & & & \\
\hline & Corregimiento 70 & 17.83 & 0 & 13.3 & 2.81 & 63.3 & & & & & & & \\
\hline & Corregimiento 90 & 4.42 & 0.03 & 2.6 & 1.03 & 6.77 & & & & & & & \\
\hline & Mayo & 7.2 & 0.007 & 1.8 & 1.16 & 2.78 & & \multirow{4}{*}{ Estratos 3} & \multirow{4}{*}{7.43} & \multirow{4}{*}{0} & \multirow{4}{*}{0.3} & \multirow{4}{*}{0.14} & \multirow{4}{*}{0.76} \\
\hline & Octubre & 5.25 & 0.02 & 2.03 & 1.09 & 3.78 & & & & & & & \\
\hline & Noviembre & 5.37 & 0.02 & 0.3 & 0.08 & 0.88 & & & & & & & \\
\hline & Sector Rural & 6.8 & 0 & 1.7 & 1.13 & 2.57 & & & & & & & \\
\hline \multirow{8}{*}{ Ancylostomideos } & Sexo Hembras & 20.39 & 0 & 0.5 & 0.37 & 0.68 & \multirow{8}{*}{ Ancylostomideos } & \multirow{8}{*}{ Comuna 13} & \multirow{8}{*}{6.37} & \multirow{8}{*}{0.01} & \multirow{8}{*}{4.05} & \multirow{8}{*}{1.26} & \multirow{8}{*}{13.03} \\
\hline & Estrato 2 & 5.06 & 0.02 & 1.4 & 1.04 & 1.92 & & & & & & & \\
\hline & Estrato 3 & 4.63 & 0.03 & 0.7 & 0.46 & 0.96 & & & & & & & \\
\hline & Comuna 1 & 10.09 & 0 & 2.5 & 1.4 & 4.61 & & & & & & & \\
\hline & Comuna 4 & 4.21 & 0.04 & 0.4 & 0.19 & 0.98 & & & & & & & \\
\hline & Corregimiento 70 & 21.49 & 0 & 15.8 & 3.32 & 74.83 & & & & & & & \\
\hline & Mayo & 12.32 & 0 & 2.2 & 1.39 & 3.35 & & & & & & & \\
\hline & Sector Rural & 5.34 & 0.02 & 1.6 & 1.07 & 2.51 & & & & & & & \\
\hline \multirow{2}{*}{ Toxocara spp } & Edad 7-12 meses & 5.49 & 0.01 & 4.4 & 1.13 & 17.21 & \multirow{2}{*}{ Toxocara spp } & Corregimiento & 6.8 & 0 & 12.8 & 1.1 & 148.39 \\
\hline & Corregimiento 90 & 20.54 & 0 & 16.3 & 3.21 & 83.18 & & 90 & & & 12.0 & & 170.0 \\
\hline & Edad 7- 12 meses & 5.46 & 0.01 & 3.2 & 1.14 & 8.79 & & & & & & & \\
\hline D. caninum & Estrato 1 & 4.43 & 0.03 & 3.2 & 1.02 & 10.19 & D. caninum & Comuna 15 & 6 & 0 & 7 & 1 & 40 \\
\hline & Comuna 13 & 4.87 & 0.02 & 3.8 & 1.06 & 13.82 & & & & & & & \\
\hline & Edad 1-6 meses & 5.03 & 0.02 & 3.5 & 1.09 & 10.92 & & inmuna 4 & & & & & \\
\hline & Estrato 5 & 6.8 & 0 & 4.7 & 1.3 & 17.2 & & Comuna 4 & 6.68 & 0 & 6.9 & 1.27 & 37.61 \\
\hline Giardia spp & Comuna 1 & 15.56 & 0 & 7.5 & 2.32 & 24.16 & Giardia spp & Comuna 7 & 507 & 002 & 56 & 104 & 30.24 \\
\hline & Comuna 11 & 7.02 & 0 & 4.9 & 1.33 & 17.59 & & 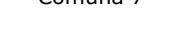 & ( & 0.02 & 5.0 & 1.07 & 00.27 \\
\hline & Diciembre & 7.27 & 0.007 & 3.9 & 1.34 & 11.58 & & Comuna 13 & 18.65 & 0 & 14.3 & 3 & 67.97 \\
\hline Trichuris spp & Corregimiento 70 & 58.29 & 0 & 52.9 & 8.9 & 314.08 & Trichuris spp & & & $18 e^{2}$ & jión & & \\
\hline 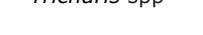 & Sector Rural & 14.81 & 0 & 10.8 & 2.39 & 48.91 & & & & & & & \\
\hline
\end{tabular}

Nota: entiéndase Factor de riesgo como un $p \leq 0.05$ y $O R \geq 1$ o Factor protector como un $p \leq 0.05$ y $O R \leq 1$.

Tabla 4. Frecuencia de seropositividad a B. canis, Leptospira sp., y T. gondii por comuna.

\begin{tabular}{ccccc}
\hline Comuna & $\begin{array}{c}\text { B. canis } \\
(\mathbf{n = 4 9 7})\end{array}$ & $\begin{array}{c}\text { Leptospira sp. } \\
(\mathbf{n}=\mathbf{5 0 0})\end{array}$ & \multicolumn{2}{c}{ T. gondii $(\mathbf{n = 5 0 0 )}$} \\
\hline 1 & 4 & 9 & SE & SPL \\
2 & 11 & 11 & 41 & 12 \\
3 & 6 & 5 & 34 & 10 \\
8 & 1 & 12 & 51 & 14 \\
13 & 14 & 8 & 52 & 6 \\
\hline n. positivos & 36 & 45 & 232 & 49 \\
$\begin{array}{c}\text { Prevalencia } \\
\text { Total (\%) }\end{array}$ & 7.2 & 9.0 & 46.4 & 9.8 \\
\hline
\end{tabular}

SE: Seropositividad Expuestos ( $\geq 1: 20-\leq 1: 160)$; SPL: Seropositividad Portadores latentes $(\geq 1 / 320)$
Tabla 5. Frecuencia de seropositividad para los diferentes serovares de Leptospira interrogans en caninos.

\begin{tabular}{ccc}
\hline Serovar Leptospira sp. & $\begin{array}{c}\text { N. Individuos } \\
\text { positivos }\end{array}$ & Prevalencia (\%) \\
\hline L. canicola & 14 & 2.8 \\
L. icterohaemorrhagiae & 10 & 2.0 \\
L. grippotyphosa & 5 & 1.0 \\
L. pomona & 4 & 0.8 \\
L. ballum & 5 & 1.0 \\
L. autumnalis & 5 & 0.1 \\
L. bratislava & 5 & 1.0 \\
L. tarassovi & 1 & 0.2 \\
\hline
\end{tabular}


Tabla 6. Análisis bivariado de los factores asociados a la seropositividad a B. canis, Leptospira sp., y T. gondii. Evidenciando factores de riesgo o protección.

\begin{tabular}{|c|c|c|c|c|c|c|}
\hline \multicolumn{7}{|c|}{ Análisis bivariado de los factores asociados a la seropositividad a $B$. canis } \\
\hline Variable dependiente & Variable independiente & $x^{2}$ & $\mathbf{p}$ & OR & IC inf & IC sup \\
\hline \multirow{3}{*}{ B. canis } & Comuna 13 & 8.6 & 0 & 2.8 & 1.37 & 5.68 \\
\hline & Comuna 8 & 7.19 & 0 & 0.1 & 0.01 & 0.77 \\
\hline & Octubre & 22.2 & 0.03 & 4 & 1.24 & 12.9 \\
\hline \multicolumn{7}{|c|}{ Análisis bivariado de los factores asociados a la seropositividad a $L$. interrogans y sus serovares } \\
\hline Variable dependiente & Variable independiente & $\mathbf{x}^{2}$ & $\mathbf{p}$ & OR & IC inf & IC sup \\
\hline Leptospira sp. & Octubre & 8 & 0.04 & 4.3 & 1.4 & 12.5 \\
\hline L. canicola & Comuna 8 & 10.5 & 0.03 & 4.2 & 1.44 & 12.34 \\
\hline \multirow{2}{*}{ L. gripppthyphosa } & Comuna 2 & 8.1 & 0.04 & 6.1 & 1.01 & 37.34 \\
\hline & Octubre & 21.84 & 0.03 & 17 & 2.7 & 112 \\
\hline L. ballum & Diciembre & 12.74 & 0.03 & 11 & 1.1 & 106 \\
\hline \multicolumn{7}{|c|}{ Análisis bivariado de los factores asociados a la seropositividad a $T$. gondii en títulos $\geq$ a $1 / 320$} \\
\hline Variable dependiente & Var Independiente & $\mathbf{X}^{2}$ & $\mathbf{p}$ & OR & IC inf & IC sup \\
\hline \multirow{2}{*}{ T. gondii } & Edad 1-6 meses & 36.79 & 0 & 7.2 & 1.16 & 44.2 \\
\hline & Estrato 1 & 9.57 & 0.02 & 2 & 1.1 & 3.74 \\
\hline
\end{tabular}

Nota: entiéndase Factor de riesgo como un $\mathrm{p} \leq 0.05$ y $\mathrm{OR} \geq 1$ o Factor protector como un $\mathrm{p} \leq 0.05$ y $\mathrm{OR} \leq 1$.

\section{DISCUSIÓN}

Parásitos gastrointestinales. Es importante resaltar que los caninos y felinos en todo Medellín son positivos a uno o varios agentes, lo que los convierte en diseminadores de parásitos y en un riesgo para la salud pública, debido a los hábitos entre humanos y mascotas. Además, representan una fuente de contaminación ambiental.

La frecuencia de positividad tanto en caninos (23.6\%) como en felinos (16.3\%) se asemeja en los primeros con la reportada en Colombia $(19.7-88.6 \%)(\underline{1}, \underline{5}, \underline{14})$ y en los segundos es menor a la reportada anteriormente en el país $(35-42.1 \%)(\underline{2}, \underline{5})$. Este estudio fue realizado en animales con dueño, explicando esto las bajas prevalencias en ambas especies; adicionalmente, en Colombia se ha evidenciado un incremento significativo en caninos callejeros, en donde los Ancylostomideos son los prevalentes $(\underline{1}, \underline{15})$.

El agente prevalente en caninos fueron los Ancylostomideos $(20.7 \%)$, parásitos que producen en humanos LMC; su prevalencia coincide con lo encontrado por otros autores en Colombia $(12.6-86.8 \%)(\underline{1}, \underline{5}, 14)$ y Latinoamérica (20$73.8 \%)(\underline{3}, \underline{16})$. Este agente se ha encontrado en zonas de esparcimiento para el humano y sus mascotas (14). En felinos la prevalencia es inferior $(5.4 \%)$ a las reportadas en Colombia (7.4\%) y Latinoamérica $(32.6-80 \%)(\underline{2}, 16)$. Además se evidenció que estos eran menos susceptibles a la afección por Ancylostomideos en comparación con los caninos, posiblemente por tener una mayor restricción en las salidas y ellos realizan sus necesidades fisiológicas en interior del hogar, accediendo en menor medida a fuentes de reinfección donde podrían infectar al humano o infectarse entre la misma especie.

En Colombia se había reportado en felinos una mayor prevalencia para Toxocara spp. (37.2\%) y Giardia spp. $(10 \%)(\underline{2}, \underline{5})$; sin embargo, es importante anotar que $D$. caninum representó un factor de riesgo en los felinos $(p=0 ; O R=4.9)$ y éstos evidenciaron una mayor prevalencia que los caninos $(7.0 \%$ vs. $1.5 \%$, respectivamente).

El Centro de Prevención y Control de Enfermedades (CDC) resalta la importancia que juega la pulga en el proceso de transmisión del $D$. caninum, debido a que al encontrarse esta infectada por los cisticercoides de $D$. caninum y ser ingerida por el humano o la mascota, puede generar el desarrollo de la enfermedad; es posible que el hábito de acicalamiento de los félidos los exponga a un mayor riesgo ante esta parasitosis por el consumo de la pulga. La infección afecta principalmente a niños en edades lactantes y preescolares con un estrecho contacto con mascotas, desarrollando cuadros digestivos que pueden comprometer su bienestar (17). Las infestaciones por pulgas deben ser controladas para asegurar un plan de prevención completo, tanto para el animal como para el humano. En otro estudio también se ha evidenciado mayor prevalencia para $D$. caninum en felinos $(6.9 \%)$ que en caninos $(2.2 \%)(\underline{3}, 14)$. Internacionalmente las prevalencias varían para caninos y felinos (1-88.3\% vs. $2.8-81.6 \%$, respectivamente) $(2,3)$ y son mayores a las reportadas nacionalmente en ambas especies (1.1-1.6\% vs. $0 \%$, respectivamente) $(\underline{1}, \underline{3}, \underline{14})$.

LMV y LMO son enfermedades causadas por Toxocara spp. $(\underline{2}, \underline{3})$. A pesar de haber presentado prevalencias en caninos $(0.9 \%)$ y felinos $(4.0 \%)$ inferiores a las reportadas en estudios previos en Colombia (2.5$11.8 \%$ vs. $5-37.2 \%$, respectivamente) $(\underline{1}, \underline{2}, \underline{5}, \underline{14})$ y otros países (11-99.4\% vs. $10 \%$, respectivamente) $(\underline{3}, \underline{14}, \underline{18})$, es un agente de gran importancia para la salud pública. En Colombia, la prevalencia en humanos es del 3.8-91\%, con seroprevalencias en Medellín del $63.3 \%$ en 30 individuos y en Latinoamérica entre 2.5 $63.2 \%(\underline{18}, 19)$. El humano puede llegar a infectarse debido a que un perro elimina al ambiente en promedio 1.4 millones de huevos al día contaminando los parque públicos y zonas de esparcimiento ( $\underline{3})$, o por la presencia de huevos infectivos en el pelaje de las mascotas (20). Los gatos también representaron un factor de riesgo $(p=0 ; O R=4.4)$ ante la toxocariasis, posiblemente ésto 
se relaciona con la presencia de huevos larvados en el pelaje y su hábito de acicalamiento, o por el consumo de huéspedes paraténicos $(14,19,20)$. El Centro de Seguridad Alimentaria y Salud Pública (CSFPH) de los Estados Unidos, resalta la importancia de la transmisión en caninos y felinos por vía transparentaría, lactogénica y por el consumo de huéspedes paraténicos que albergan la fase larvaria de Toxocara spp. en sus tejidos.

Aunque no se realizó genotipificación de Giardia spp. y no se determinó si el protozoario encontrado pertenece a los genotipos de infección cruzada o especie específicos de $G$. duodenalis ( $\underline{5})$, es interesante tener en cuenta que aunque la prevalencia encontrada para caninos y felinos es baja en comparación con lo reportado anteriormente en Colombia $(0.8 \%-16 \%$ vs. $14.8 \%$, respectivamente) $(\underline{2}, \underline{3}, \underline{14})$ y otros países $(10-35 \%$ vs. $10-15 \%$, respectivamente) $(\underline{3}, 14)$, éste agente produce cuadros de gastroenteritis en niños menores de cinco años, con prevalencias en personas del $13 \%$ y hasta del $33 \%$ en países en desarrollo $(\underline{3}, \underline{5})$.

Los resultados pueden deberse a que el agua que abastece los hogares de la zona urbana de Medellín es potable y que las condiciones higiénico sanitarias de la ciudad son buenas debido a que cuenta con alcantarillado para las aguas negras, lo cual disminuye el riesgo de infección cruzada; siendo esto respaldado por la CDC y la CSFPH que reflejan la importancia del consumo de agua potable para disminuir el riesgo de transmisión de Giardia spp. En contraste, este agente fue el único que mostró una mayor susceptibilidad en aquellos individuos que habitan en un estrato socioeconómico alto, esto puede estar influenciado por el acceso a fuentes de reinfección ubicadas a las afueras del casco urbano de la ciudad de Medellín, como fincas o pueblos donde aún no se cuente con un riego de agua potable o al acceso de aguas residuales, ríos, lagos o charcos en zonas de esparcimiento fuera de la ciudad; ya que los caninos o felinos ubicados en estos estratos tienen mayor posibilidad de ser trasladados junto al núcleo familiar a otros puntos donde puede llegar a aumentar el riesgo de reinfección $(\underline{3}, \underline{5})$.

D. caninum y los Ancylostomideos evidenciaron un factor de riesgo en los estratos socioeconómicos bajos, lo cual puede relacionarse con un menor acceso a los servicios veterinarios básicos y al hábito semidoméstico de las mascotas, frecuente en estos estratos, que contribuye a una mayor exposición $(\underline{3}, \underline{5})$.

La edad como factor de riesgo encontrada para Toxocara spp. difiere de lo reportado en otros estudios, los cuales indican una mayor prevalencia en los cachorros menores a 6 meses, ya que el principal mecanismo de trasmisión de este parasito es por vía lactogénica y placentaria $(1,3,14)$. Los resultados encontrados en el presente estudio podrían indicar una posible reinfección o una ausencia de vermifugación en los individuos analizados $(\underline{14}, \underline{20})$.

En el estudio de Sierra et al (1) , también se evidenció en caninos una mayor prevalencia a Ancylostomideos en el sector rural de Antioquia (87.1\%) que en los pertenecientes al urbano (24.1\%). Para Toxocara spp., se reporta mayor contaminación de suelos en zonas urbanas en comparación con los suelos de zonas sub-urbanas o rurales (19), siendo ésto contrario a lo encontrado en el corregimiento 90. Sin embargo, para Trichuris spp. no existen reportes en la literatura que respalde el factor de riesgo hallado en el sector rural.

En el presente estudio los meses secos representaron un factor de riesgo para la presencia de $D$. caninum $(p=0.01 ; O R=10.7)$ y Giardia spp. $(p=0.007 ; O R=3.9)$, relacionándose con lo encontrado en Bolivia, donde para ambos agentes se evidenció una mayor prevalencia en temporada seca $(\underline{3}, \underline{14}, \underline{17})$; mientras que los meses húmedos reflejaron asociación para la positividad a Ancylostomideos $(p=0 ; O R=2.2)$ y es respaldado por la literatura $(\underline{16}, \underline{17})$.

Brucella canis. Fue reportada en Colombia desde 2009; adicionalmente, Castrillón et al $(\underline{10})$ establecieron que la seropositividad en humanos convivientes con caninos destinados a la cría era del $17 \%(\underline{7}, \underline{10})$. La prevalencia del $7.2 \%$ corresponden en su mayoría a caninos sin esterilizar y sin aparente sintomatología. La comuna más seropositiva y donde se halló un factor de riesgo, corresponde a aquella donde más se evidencian perros sin domicilio estable o domésticos-callejero acorde con información brindada por los habitantes de la zona. Los meses del año con mayor seropositividad corresponden a los meses con mayor pluviosidad en la región y representaron un factor de riesgo para la seropositividad, posiblemente porque la lluvia favorece la permanencia del agente en materia orgánica, retrasando su desecación y aumentando la probabilidad de transmisión por fómites e ingesta de material contaminado (10).

Leptospira sp. En Colombia la seroprevalencia en el hombre se ha reportado entre $12-62 \%$ (21). Es un problema de salud pública que emergió en las áreas urbanas por el crecimiento de las ciudades, suburbios, desplazamiento y zonas de invasión $(\underline{22}, \underline{23})$, donde la falta de saneamiento básico, las altas precipitaciones, las altas infestaciones de roedores y la presencia de perros callejeros favorece la transmisión $(\underline{21}, \underline{22})$. Ciudades en Colombia han reportado una seroprevalencia canina a Leptospira sp., del $45 \%$ en periodos pasados, adicional al reporte de transmisión de leptospirosis de canino infectado a humano (22).

En Medellín la presencia de roedores implica diferentes esfuerzos por parte de las entidades de salud ambiental para establecer los niveles de infestación, las medidas de prevención y control respectivas. Teniendo en cuenta la situación actual frente a la presencia de roedores en todo Medellín y sus implicaciones sanitarias (ㅁ), sumada a la seropositividad encontrada para diferentes serovares de Leptospira interrogans en el estudio y a la notificación de Antioquia como el departamento con más casos humanos de leptospirosis confirmados en el país, reportado por el Sivigila (22); tanto en caninos como en humanos los serovares más reportados son canicola e icterohaemorrhagiae, lo que hace posible pensar en una infección interespecie. Adicionalmente, los roedores son los reservorios por excelencia de $L$. icterohaemorrhagiae y $L$. ballum, reportadas en diferentes comunas.

Los caninos pueden cursar la enfermedad de forma asintomática, excretando grandes cantidades de Leptospira durante meses o años postinfección (24). Adicionalmente con Leptospira sp., se ha visto relación con zonas inundables o de alta humedad (ㅁ); en estas condiciones sobrevive hasta 350 días (24) y puede 
infectar al hombre, a perros susceptibles y a los roedores, perpetuando así el ciclo de transmisión $(\underline{8}, \underline{24})$. Esto puede explicar a octubre (periodo de lluvias) como factor de riesgo para Leptospira sp. y L. gripppthyphosa $(\underline{8}, \underline{21}, \underline{22})$, dados los hallazgos es necesario implementar planes vacunales en los caninos para evitar su contagio y posterior transmisión al humano (ㅁ).

Los hallazgos en este estudio pueden deberse a las falencias o a la mala implementación del plan de prevención vacunal, pues los únicos serovares incluidos en el esquema canino son Canicola e Icterohaemorrhagiae $(\underline{25})$, posiblemente debido al bajo nivel sociéconomico, a la baja posibilidad económica para acceder a las vacunas o por desconocimiento poblacional. Igualmente queda abierta la probabilidad de que los caninos estén expuestos a otras especies domésticas o silvestres transmisoras de diferentes serovares que carecen de vacunación y en las cuales se han reportado dichos serovares poco frecuentes $(\underline{8}, \underline{25})$.

Toxoplasma gondii. Entre el $50-60 \%$ de las mujeres embarazadas en Colombia presentan anticuerpos antitoxoplasma (9), lo que indica una alta circulación del agente en el medio; por sus condiciones tropicales, se considera la toxoplasmosis en Colombia una enfermedad vigente y de serias repercusiones sobre las mujeres gestantes y los neonatos $(\underline{9}, \underline{13})$. Existe un riesgo potencial de que los felinos con elevados títulos de anticuerpos ( $\geq 1: 320)$ puedan en algún momento salir del periodo de latencia e iniciar la eliminación de quistes de $T$. gondii, considerándose esta es una de las muchas causas de transmisión y lo que explicaría los altos porcentajes de mujeres seropositivas al agente $(\underline{9}, \underline{26})$. Aunque el felino es el hospedero definitivo, en el cual se desarrolla el ciclo sexual del parásito $(\underline{9}, \underline{13})$; se debe tener presente que las formas de transmisión al humano no requieren del contacto directo con éste (9). Pese a la alta seropositividad encontrada, no hubo felinos con infección activa durante el muestreo.

La seropositividad en felinos del estrato socioeconómico 1 , el de menor calidad de vida, puede estar asociado al poco acceso a agua potable tanto para la población como para los animales (22). El riesgo que presentan los gatos entre 1-6 meses puede deberse a la transmisión transplacentaria y lactogénica de la madre a sus crías (27); los títulos en adultos, indican exposición previa más no infección vigente o latencia y podrían deberse a las poblaciones de felinos ferales, población en la que a nivel mundial se ha reportado mayor seropositividad $(\underline{9}, \underline{13}, \underline{26})$.

Comentarios finales. Este trabajo demuestra la importancia de variables como el estrato socioeconómico y la zona de muestreo, sobre la positividad a diferentes agentes de origen zoonótico de alto y mediano riesgo. Es importante hacer visible la problemática de zoonosis en Colombia y su riesgo para la salud pública. Medellín, aunque ha sido pionera en temas de bienestar animal, debe establecer planes sanitarios pertinentes para la desparasitación y vacunación de animales de compañía.

\section{Conflictos de interés}

Los autores informan no tener ningún conflicto de interés.

\section{Agradecimientos}

Universidad de Antioquia, Grupo de Investigación Biogénesis. Secretaria de Salud del Municipio de Medellín.

\section{REFERENCIAS}

1. Sierra $V$, Jiménez JD, Alzate AA, Cardona JA, Ríos LA. Prevalencia de parásitos intestinales en perros de dos centros de bienestar animal de Medellín y el oriente antioque-o (Colombia), 2014. Rev Med Vet. 2015; (30):55-66. DOI: https://doi.org/10.19052/ $\underline{\mathrm{mv} .3609}$

2. Echeverry DM, Giraldo MI, Casta-o JC. Prevalence of intestinal helminths in cats in Quindío, Colombia. Biomédica. 2012; 32(3):430-436. DOI: https://doi. org/10.7705/biomedica.v32i3.439 PMid:23715191

3. González AC, Giraldo JC. Prevalencia de parásitos intestinales zoonóticos en caninos (Canis Lupus Familiaris) del área urbana del municipio de Coyaima (Tolima). Revista Med. 2015;23(2):24-34. DOI: https://doi.org/10.18359/rmed.1743

4. Alcaldía de Medellín. Resultados Encuesta de Calidad de Vida 2015 [Internet]. Medellín: Alcaldía de Medellín; 2016 [cited 2016 Jul 30]. Available from: https://www.medellin.gov.co/irj/portal/medellin.
5. Rodríguez VE, Espinosa O, Carranza JC, Arévalo A, Vallejo GA. Detección de parásitos intestinales en niňos preescolares y animales domésticos del municipio de Ibagué (Tolima). RCCA. 2015; 7(1):8.

6. Manini MP, Marchioro AA, Colli CM, Nishi L, Falavigna AL. Association between contamination of public squares and seropositivity for Toxocara spp. in children. Vet Parasitol. 2012; 188(1):48-52. DOI: https://doi.org/10.1016/j.vetpar.2012.03.011 PMid:22480882

7. Sánchez $M M$, Ortiz LF, Castrillón LL, Giraldo $C A$, Olivera M. Application of a polymerase chain reaction test for the detection of Brucella canis from clinical samples of canines and humans. Rev Colomb Ciencias Pecu. 2014; 27(1):3-11.

8. Raghavan R, Brenner K, Higgins J, Hutchinson JS, Harkin K. Evaluations of hydrologic risk factors for canine leptospirosis: 94 cases (20022009). Prev Vet Med. 2012; 107(1):105-109. https://doi.org/10.1016/j.prevetmed.2012.05.004 PMid:22676955 
9. Palmezano JM, Plazas LK, Rojas D. Infección por toxoplasma: panorama actual. Spei Domus. 2015; 11(22):47-56. https://doi.org/10.16925/ sp.v11i22.1154

10. Castrillón L, Giraldo CA, Sánchez MM, Olivera M. Factors associated with Brucella canis seropositivity in kennels of two regions of Antioquia, Colombia. Cad Saude Publica. 2013; 29(10):1955-1973. DOI: http://dx.doi.org/10.1590/0102-311X00133013 PMID: 24127092

11. Restrepo A. Guía en Parasitología Veterinaria. 1st ed. Colombia: Exitodinamica Editores; 1983.

12. Haddadzadeh $H$, Khazraiinia $P$, Aslani $M$, Rezaeian M, Jamshidi S, Taheri M, et al. Seroprevalence of Toxoplasma gondii infection in stray and household cats in Tehran. Vet Parasitol. 2006; 138(3):211-216. DOI: https://doi.org/10.1016/j.vetpar.2006.02.010 PMid: 16529863

13. Palmero ML, Carballés V. Enfermedades Infecciosas Felinas. 1st ed. Espa-a: Editorial Servet; 2010.

14. Alarcón Z, Juyo V, Larrotta J. Caracterización epidemiológica de parásitos gastrointestinales zoonóticos en caninos con due-o del área urbana del municipio de La mesa, Cundinamarca. Rev Med Vet Zoot. 2015; 62(1):20-36. DOI: https://doi. org/10.15446/rfmvz.v62n1.49382

15. Solarte LD, Casta-eda R, Pulido AdP. Parásitos gastrointestinales en perros callejeros del centro de zoonosis de Bogotá DC, Colombia. Neotrop Helminthol. 2013; 7(1):83-93. URL: http://sisbib. unmsm.edu.pe/bvrevistas/neohel/v7n1/pdf/ a09v7n1.pdf

16. Plascencia A, Proy $\mathrm{H}$, Eljure N, Atoche C, Calderón C. Larva migrans cutánea relacionada con Ancylostomas. Dermatol Rev Mex. 2013; 57(6):454460. URL: http://www.medigraphic.com/pdfs/ derrevmex/rmd-2013/rmd136g.pdf

17. Llanos M, Condori M, Ibá-ez T, Loza-Murguia M. Parasitósis entérica en caninos (Canis familiaris) en el área urbana de Coroico, Nor Yungas Departamento de La Paz, Bolivia. J Selva Andina Res Soc. 2010; 1(1):37-49. URL: http://ucbconocimiento.ucbcba. edu.bo/index.php/JSARS/article/view/130

18. Olave AM, Mesa JA, Botero JH, Pati-o EB, García GM, Alzate JF. Production and evaluation of the recombinant antigen TES-30 of Toxocara canis for the immunodiagnosis of toxocariasis. Biomédica. 2016; 36(1):39-51. DOI: https://doi.org/10.7705/ biomedica.v36i1.2617 PMid:27622437
19. Bre-a JP, Hernández R, Hernández A, Castaeda R, Espinoza Y, Roldán W, et al. Toxocariosis humana en el Perú: aspectos epidemiológicos, clínicos y de laboratorio. Acta Méd Peruana. 2011; 28(4):228-236. URL: http://www. scielo.org.pe/scielo.php?script $=$ sci_arttext\&pid $=\mathrm{S} 1728-59172011000400010$

20. Wolfe A, Wright I. Human toxocariasis and direct contact with dogs. Vet Rec. 2003; 152(14):419421. DOI: https://doi.org/10.1136/vr.152.14.419 PMid: 12708589

21. Dechner A. A retrospective analysis of the leptospirosis research in Colombia. J Infect Dev Ctries. 2014; 8(03):258-264. DOI: https://doi. org/10.3855/jidc.3123 PMid:24619254

22. Escandón K, Osorio L, Astudillo M. Seroprevalence and factors associated with Leptospira infection in an urban district of Cali, Colombia. Cad Saude Publica. 2017; 33(5):e00039216. DOI: https://doi. org/10.1590/0102-311×00039216

23. Sarkar U, Nascimento SF, Barbosa R, Martins R, Nuevo H, Kalafanos I, et al. Population-based casecontrol investigation of risk factors for leptospirosis during an urban epidemic. Am J Trop Med Hyg. 2002; 66(5):605-610. DOI: https://doi.org/10.4269/ ajtmh.2002.66.605 PMid: 12201599

24. Faine S, Adler B, Bolin C, Perolat P. Leptospira and leptospirosis 1999-2000. 2nd ed. Melbourne: MediSci; 2000.

25. Medrano C, Díaz CA, Dalmau EA. Diagnóstico de leptospirosis canina por medio de las técnicas Dot-ELISA y MAT en perros con enfermedad renal en Bogotá. Rev Med Vet. 2011; 21:133-145. DOI: https://doi.org/10.19052/mv.568

26. Ca-ón WA, López N, Gómez JE, Dubey JP. An overview of seventy years of research (1944-2014) on toxoplasmosis in Colombia, South America. Parasit Vectors. 2014; 7(1):427. DOI: https://doi. org/10.1186/1756-3305-7-427 PMid:25190525

27. Yongzhen P, Xuehui J, Changguo L, Shujing G. Dynamics of a model of Toxoplasmosis disease in cat and human with varying size populations. Mathematics and Computers in Simulation. 2018; 144(C):52-59. DOI: https://doi.org/10.1016/j. matcom.2017.06.007 